\title{
An Economic Method for the Solution of the Scalar Wave Equation for Arbitrarily Shaped Optical Waveguides
}

\author{
HUGO J. W. M. HOEKSTRA
}

\begin{abstract}
In the method presented here, the discrete sine method, the basis functions consist of sine functions defined on a set of paralle discretization lines. The method is a combination of a scalar version of the finite difference method and the sine method. The choice of the basis set leads, for the eigenvalue equation to be solved, to a sparse matrix with a small bandwidth. As a consequence, the propagation constant of guided modes in optical waveguides may be calculated with short computation times and low storage needs. Results, obtained with the method, for three different waveguiding structures, are compared with those of other methods.
\end{abstract}

\section{INTRODUCTION}

$\mathrm{F}^{\mathrm{o}}$ OR APPLICATIONS in integrated optics a large variety of channel waveguides are used in order to get two-dimensional confinement of the light (see, e.g., [1] and $[1$, references]). For modeling and testing of these waveguiding structures, reliable computational methods are needed. The wave equation for two-dimensional waveguides cannot be solved analytically. Approximate computational methods, both scalar and vectorial, have been the subject of many papers (see [1], [2], [1, references], [2, references], and [3]).

In this paper we present a new method, the discrete sine method (DSM), which may be considered as a combination of a scalar version of the finite difference method (SFDM) and the sine method (SM) [4]. For the finite difference method (FDM), the reader is referred to [1], [5] The theory of the DSM, its merits, and its limitations are discussed in detail in Section II. Computational results of the method and those of other methods are presented in Section III.

\section{THEORY}

We consider a $z$-invariant wave guiding structure. A mode is defined as a field solution of Maxwell's equations of the form

$$
\begin{aligned}
& \boldsymbol{E}(x, y, z)=\boldsymbol{e}(x, y) \exp (-i \beta z) \\
& \boldsymbol{H}(x, y, z)=\boldsymbol{h}(x, y) \exp (-i \beta z)
\end{aligned}
$$

Manuscript received September 19, 1989; revised December 1, 1989 The author is with the University of Twente, Enschede, The Netherlands

IEEE Log Number 8933952. for the electric and magnetic field, respectively. The time dependence exp ( $i \omega t)$ has been suppressed. Maxwell's equations for the modal fields $\boldsymbol{e}$ and $\boldsymbol{h}$ have been given by Snyder and Love [6]. The vectorial wave equation may be expressed in terms of two modal field components: $\boldsymbol{h}$ or $e_{t}$ or $h_{z}$ and $e_{z}$, where the subscript $t$ indicates the transverse field components. For its relative simplicity, and also to circumvent the occurrence of spurious modes [1], we use the equations containing the transverse magnetic field:

$$
\left\{\Delta_{t}+n^{2} k^{2}-\beta^{2}\right\} \boldsymbol{h}_{t}=\left(\nabla_{t} \times \boldsymbol{h}_{t}\right) \times \nabla_{t} \ln \left(n^{2}\right) .
$$

Here,

$$
\begin{aligned}
\Delta_{t} & =\frac{\partial^{2}}{\partial x^{2}}+\frac{\partial^{2}}{\partial y^{2}}, \\
\nabla_{t} \times \boldsymbol{h}_{t} & =z\left(\frac{\partial h_{y}}{\partial x}-\frac{\partial h_{x}}{\partial y}\right), \text { and } n=n(x, y) .
\end{aligned}
$$

Inspection of (2) shows that the right-hand side is nonvanishing only at the interfaces. The term takes into account the boundary conditions in operator form.

The method we propose takes advantage of both the sparseness of the matrices, occurring in the (S)FDM and the (often) rapid convergence, as a function of the number of basis functions, in the SM. Furthermore, the fact that the operator $\Delta_{t}$ is diagonal, when operating on sine functions, often leads to very sparse matrices, as is demonstrated in the next section. In all of the three methods mentioned above, the waveguiding structure is enclosed by a rectangle, which should be chosen large enough so that the eigenfunctions, corresponding to guided modes, may safely be assumed to be negligible on its boundary. In the (S)FDM the size of the box may be chosen smaller by approximating the wave function outside the box by exponentially decaying basis functions [5]. The eigenfunctions are written as a linear combination of a (finite) orthonormal basis set; which allows the eigenvalue equation (2) to be written as a matrix eigenvalue equation. First we give a short discussion on the FDM and the SM.

The FDM consists of dividing the rectangle, which encloses the structure, into a (graded) mesh. The bases functions are discretisized and are defined on the nodal points of the mesh. The second-order differential operators, oc- 
curring in (2), are rewritten as five-point operators [1], [4]. We remark that, for the FDM, appreciable computation speed (or accuracy) may be gained in the following ways.

First, if the mesh size is varied, the corresponding eigenvalues $\beta^{2}$ behave as [7]

$$
\beta^{2}(w)=\beta^{2}(0)+O\left(w^{2}\right) .
$$

Here $w$ is a measure for the mesh size, which should be varied in a uniform way, i.e., without changing the relative distances of the mesh over the waveguide structure. The inaccuracy of the calculated eigenvalues is due to the finite mesh size, i.e., due to approximating the secondorder differential operators by difference operators. The consequence of (4) is that the calculated eigenvalues $\beta^{2}(w)$ may be extrapolated to $\beta^{2}(0)$, if the used values of $w$ are small enough to neglect third and higher order terms. As the number of operations needed to solve matrix eigenvalue problems grows rapidly as a function of the number of basis functions, we may gain appreciable speed this way. The relation between $\beta^{2}$ and $w$, as given by (4), is demonstrated by an example in the next section; see also the results given in [1, Fig. 3]. An alternative for the above may be used for a uniform square mesh and consists of replacing the five-point difference operator by a suitably chosen, nine-point difference operator [7].

The second suggestion holds for symmetric structures. Here the eigenfunctions will reflect that symmetry; so the basis set can be subdivided into subsets. Group theory gives full account of this problem. In practical situations we often have to do with waveguides having a mirror plane. Then the subsets may be chosen to consist of even and odd basis functions. For the (S)FDM these are of the form

$$
\left(\boldsymbol{h}_{p}+\boldsymbol{h}_{p^{\prime}}\right) / \sqrt{2} \quad \text { and } \quad\left(\boldsymbol{h}_{p}-\boldsymbol{h}_{p^{\prime}}\right) / \sqrt{2}
$$

for even and odd modes, respectively. Here $p$ and $p^{\prime}$ denote points which coincide on reflection across the mirror plane.

In the SM the basis functions are products of sine functions, which form an orthonormal set and vanish at the boundaries of the enclosing rectangle, i.e., at $x=0, L_{x}$ and $y=0, L_{y}$. The set is given by [4]

$$
\phi_{n, m}=\frac{2}{\sqrt{L_{x} L_{y}}} \sin \left(n \pi x / L_{x}\right) \cdot \sin \left(m \pi y / L_{y}\right) .
$$

Here $m$ and $n$ are natural numbers greater than zero. Equation (6) represents even or odd functions with respect to the $x$ axis for $n$ is odd or even, respectively. Note that the functions of the basis set and the corresponding derivatives are continuous. As, thus, the interface conditions of the (vectorial) wave equation cannot be met, this makes the set suitable to solve the scalar wave equation, with vanishing interface conditions, only. The number of basis functions needed for an accurate result depends strongly on the shape of the waveguide. For the SM one can use as a rule of thumb that, e.g., for the $y$ direction, the fol-

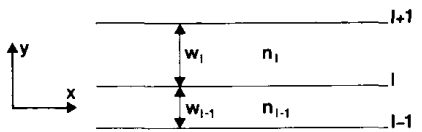

Fig. 1. Illustration of the DSM treatment.

lowing relation should hold:

$$
L_{y} / m_{\max } \ll l_{y} .
$$

Here $l_{y}$ is the smallest typical length of the structure in the $y$ direction and $m_{\max }$ is the maximum value of $m$, occurring in (6). Thus the basis set is large enough to let the eigensolutions be able to vary rapidly in the vicinity of such small details. For example, for ridge guides, $l_{y}$ is the thickness of the ridge, which may be quite small in practical situations.

In the DSM, the method presented here, the rectangle, containing the waveguide structure, is divided by generally nonequidistant discretization lines, parallel on, say, the $x$ axis. On each line a set of sine functions is defined as follows:

$$
\phi_{l, m}=\delta_{l}\left(\frac{2}{L_{x}}\right)^{1 / 2} \sin \left(\frac{m \pi x}{L_{x}}\right) .
$$

Here $\delta_{l}=1$ on the $l$ th line and zero everywhere else.

For the waveguide structure we assume that the field solutions are either pure TE or pure TM. Inspecting (2) shows that this condition is approximately fulfilled for structures with smooth index changes or for structures, such as ridge waveguides having a ridge with large width $\Delta x$ and small height $\Delta y$ for which we may put

$$
\frac{\partial h_{y}}{\partial x} \cong 0 \quad \text { and } \quad \frac{\partial \ln \left(n^{2}\right)}{\partial x} \cong 0 .
$$

The conditions (9) hold if the frequency is far from cutoff, so that the field intensity at the interfaces is relatively small. Then, the following partial differential equations hold:

$$
\begin{aligned}
\left(\Delta_{t}+n^{2} k^{2}-\beta^{2}\right) h_{y} & =0, \\
\left(\Delta_{t}+n^{2} k^{2}-\frac{\partial \ln \left(n^{2}\right)}{\partial y} \frac{\partial}{\partial y}-\beta^{2}\right) h_{x} & =0
\end{aligned}
$$

for TE and TM modes, respectively. For the basis set, given in (8), we have to replace differential operators for the $y$ direction by three-point difference operators, e.g. (see Fig. 1):

$$
\begin{aligned}
\frac{\partial^{2}}{\partial y^{2}} f_{l}(x) \cong & \frac{2 f_{l+1}(x)}{w_{l}\left(w_{l}+w_{l-1}\right)} \\
& +\frac{2 f_{l-1}(x)}{w_{l-1}\left(w_{l}+w_{l-1}\right)}-\frac{2 f_{l}(x)}{w_{l} w_{l-1}} .
\end{aligned}
$$

Using Dirac's bra and ket notation, $\langle l, m|$ and $|l, m\rangle$, respectively, for $\phi_{l, m}$, the operators for the DSM may be 


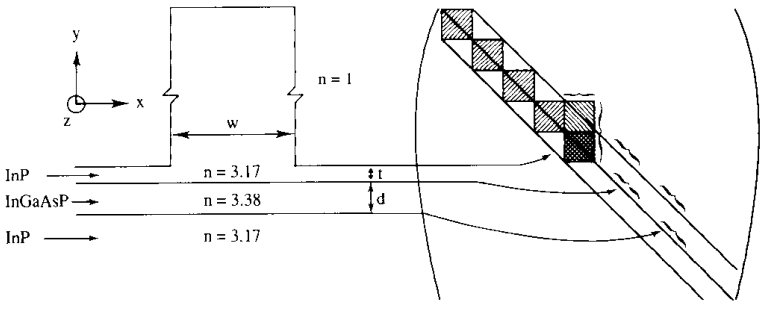

Fig. 2. Waveguiding structure and a schematic reflection of the corresponding matrix $A$ occurring in the DSM. The fat lines and the hatched part indicate nonvanishing matrix elements. The arrows connect corresponding parts of the matrix and the structure. The braces indicate the locations in the matrix containing contributions, occurring for TM modes only.

written as

$$
\begin{aligned}
& \frac{\partial^{2}}{\partial x^{2}} \rightarrow \sum_{l, m}-\left(\frac{m \pi}{L_{x}}\right)^{2}|l, m\rangle\langle l, m| \\
& \frac{\partial^{2}}{\partial y^{2}} \rightarrow \sum_{l, m}-2 \frac{|l, m\rangle\langle l, m|}{w_{l} w_{l-1}}+2 \frac{|l+1, m\rangle\langle l, m|}{w_{l}\left(w_{l}+w_{l-1}\right)} \\
&+2 \frac{|l-1, m\rangle\langle l, m|}{w_{l-1}\left(w_{l}+w_{l-1}\right)} \\
& \frac{\partial}{\ln \left(n^{2}\right)} \frac{\partial}{\partial y} \rightarrow \sum_{l, m} \\
& \sigma_{l}\{|l, m\rangle\langle l, m|-| l-1, m\rangle\langle l, m|\}
\end{aligned}
$$

with

$$
\sigma_{l}=2 \frac{n_{l}^{2}-n_{l-1}^{2}}{n_{l-1}^{2} w_{l-1}\left(w_{l}+w_{l-1}\right)}
$$

The eigenfunctions may be written as

$$
\sum_{l, m} a_{l, m}|l, m\rangle
$$

Then, using (10) and (12), we may find the matrix eigenvalue equation, which is of the form

$$
\sum_{l, m} A_{p, q: l, m} a_{l, m}=\beta^{2} a_{p, q} \text { or } \operatorname{det}\left(\boldsymbol{A}-\beta^{2} \boldsymbol{I}\right)=0
$$

Here $\boldsymbol{I}$ is the unit matrix. It is convenient to order the basis functions as $|1,1\rangle,|1,2\rangle, \cdots,\left|1, n_{\max }\right\rangle,|2,1\rangle$, ... , etc., and if the structure has a mirror plane parallel to the $y$ axis, to divide the set into subsets, containing the even and odd sine functions. For TE modes we thus obtain a sparse symmetric (except at locations in the matrix corresponding to variations in the step size $w_{l}$ of the discretization lines) band matrix with a small bandwidth, leading to short computation times. For TM modes the matrix $\boldsymbol{A}$ is due to the last operator given in (12) (partly) asymmetric and the bandwidth is twice as large as that for TE modes [except for planar structures; see the remarks below (16)]. The latter is due to the fact that, contrary to TE modes, for which

$$
A_{l, m: p . q}=0, \quad \text { if } p=l \pm 1 \text { and } m \neq q
$$

for TM modes, for some values of $l$,

$$
A_{l, m ; p, q} \neq 0, \quad \text { if } p=l+1, \quad \text { for all } m, q \text {. }
$$

Inspection of (12) shows that (16) holds only if the difference between the refractive indexes, along the lines $l$ and $p=l+1$, is not a constant as a function of $x$. Generally, this will occur only for a few values of $p$, so that, if, e.g., the method of Gaussian elimination is used, this will lead to only a small increase of the computation time, with respect to that for TE modes. If the difference $n_{l+1}^{2}$ $-n_{l}^{2}$ is a constant, then (15) holds also for TM modes. The discussion above is illustrated by Fig. 2 .

\section{Computational Results}

In Fig. 3 we compare results obtained with the DSM, SM, and the SFDM with those obtained using other methods [8], [9], [9, references], and [10]. The figure shows the propagation constant of the fundamental TE mode of the structure shown in the inset as a function of $k h$, with $k=2 \pi / \lambda$ and $h=2 \mu \mathrm{m}$. The effective index method (EIM) [8], the domain integral method (DIM) [9], and the weighted index method (WIM) [10] are introduced elsewhere.

For the calculations we enclosed the structure by a rectangle which was chosen sufficiently large; i.e., the results did not depend on variations in the lengths $L_{x}$ and $L_{y}$, in the $x$ and $y$ direction, respectively. We used the values between $L_{x}=16 \mu \mathrm{m}$ and $L_{y}=12 \mu \mathrm{m}$ for $k h=7$ and $L_{x}$ $=28 \mu \mathrm{m}$ and $L_{y}=18 \mu \mathrm{m}$ for $k h=5$.

The eigenvalue for the fundamental mode was for the SM calculated using routines from the NAG library. The eigenvalues for the DSM and the SFDM were found using Gaussian elimination, without pivoting, and the method of bisection in order to approximate the value of $\beta$ for which

$$
\operatorname{det}\left(\boldsymbol{A}-\beta^{2} \boldsymbol{I}\right)=0 .
$$

The SFDM was made more accurate by extrapolation of the results (see Fig. 4). The number of basis functions needed to obtain an accuracy in $N_{\text {eff }}$, defined by $\beta=k N_{\text {eff }}$, of $10^{-4}$, for the various methods we used and for $k h=7$, is given in Table I. Note that the computer storage needed to calculate the eigenvalues with the DSM, for this problem, is equal to the lower (or higher) triangular part of a symmetric submatrix of order 8 , i.e., 36 .

From Fig. 3 we see that the results for the DSM, the SFDM, and the SM almost coincide. The small differences are attributed to the fact that the second derivative of discrete functions may be, contrary to that of sine functions, discontinuous at the interfaces. Compared with a rigorous, vectorial method as the DIM [9], the results are quite good, even close to cutoff, where the WIM [10] seems not to be very reliable. This close agreement between various methods, both vectorial and scalar, does not always hold and depends on the waveguide structure. The results of the WIM differ appreciably from those of the other scalar methods, in this example and also in the next. This is assigned to the fact that the field solutions 


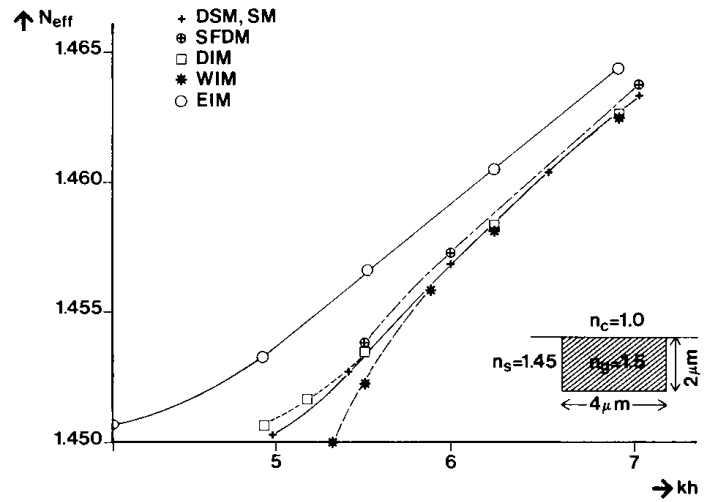

Fig. 3. Dispersion curves of the fundamental TE mode of the depicted waveguide.

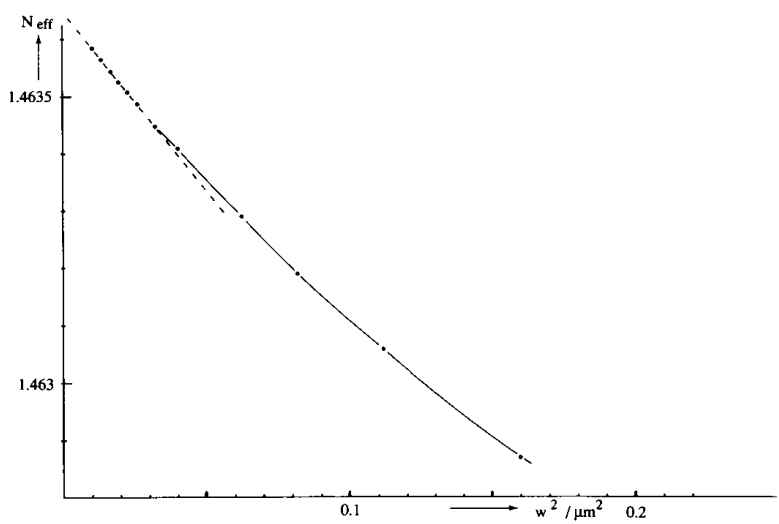

Fig. 4. The dependence of the effective refractive index $N_{\text {ef }}$ of the structure given in Fig. 3, for $k h=7$, as calculated by the SFDM, as a function of the squared mesh size $w^{2}$. For small values of $w$, the relation is approximately linear. In the calculations we have used, in order to ease the programming, the averaged squared refractive index for mesh points lying on the horizontal interfaces (see Fig. 1). The vertical interfaces are chosen in between two columns of mesh points.

TABLE I

Approximate Number of Basis Functions NeEded to Obtain an ACCURACY IN $N_{\text {eff }}$ OF $10^{-4}$, WITH THE INDICATED METHODS FOR THE FundameNTAL MODE OF WAVEguIDE OF Fig. 3, wITH $k h=7$ (We used an enclosing rectangle with dimensions $L_{x}=16$ and $L_{y}=12$ For all three methods we used only the even, with respect to the $x$ axis, basis functions)

\begin{tabular}{|l|c|c|c|c|}
\hline & & x-direction & $y$-direction & \multicolumn{2}{|c|}{ matrix A } \\
\cline { 2 - 5 } & & & order & bandwidth \\
\hline SSM* & 7 & 60 & 420 & 7 \\
SFDM** & 7 & 16 & 112 & $: 11$ \\
SFDM** $^{*}$ & $\approx 32$ & 120 & $\approx 10^{4}$ & 80 \\
& & $\approx 48$ & $\approx 1536$ & $\approx 32$ \\
\hline
\end{tabular}

- we used a set of equidistant discretization Iines

* without extrapolation (see text); square mesh.

*.*ith extrapolation; square mesh.

of this method are supposed to be separable [10], i.e., the field solutions are assumed to be of the form $h(x, y)=$ $f(x) g(y)$.

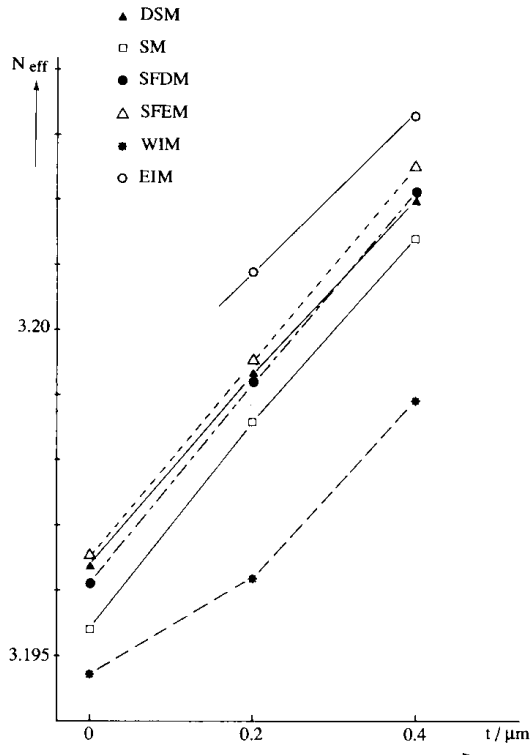

Fig. 5. The effective refractive index of the fundamental TE mode of the structure given in Fig. 2 as a function of the thickness of the InP layer $t$. The wavelength $\lambda=1.55 \mu \mathrm{m}, w=2.4 \mu \mathrm{m}$, and $d=0.2 \mu \mathrm{m}$.

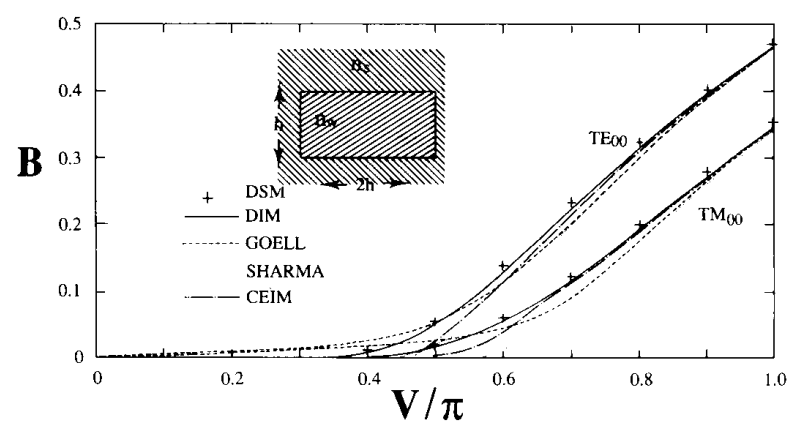

Fig. 6. Dispersion curves for an embedded waveguide structure. The normalized mode index $B$ is displayed as a function of $V / \pi$. Here $V$ is the normalized frequency (see text).

In Fig. 5 we present a part of the results presented by the COST-216 working group [2], together with results obtained with the DSM, for the fundamental mode of the waveguide structure given in Fig. 2. As in Fig. 4 we see that the effective index $N_{\text {eff }}$ calculated with the various scalar methods gives results which differ only slightly from each other. The somewhat low values obtained with the sine method is attributed to the limited basis set (see (7) and the discussion given there), i.e., 15 and 35 sine functions for the $x$ and $y$ direction, respectively.

As a final example we have used the DSM to calculate propagation constants of the structure, treated by [11][13] and depicted in Fig. 6. The structure consists of a step-index waveguide embedded in a homogeneous medium. In order to take into account the interface conditions properly, we have used (10b) for both the TE and the TM modes, choosing the $x$ axis to be perpendicular 
and parallel to the longer side of the rectangular structure, respectively. Results are presented in Fig. 6, where we have used the normalized mode-index $B$ as a function of the normalized frequency $V$ divided by $\pi$ :

$$
B=\frac{N_{\mathrm{eff}}^{2}-n_{s}^{2}}{n_{w}^{2}-n_{s}^{2}}
$$

and

$$
V=k h \sqrt{n_{w}^{2}-n_{s}^{2}}
$$

Our results agree quite well with those of the other methods, especially with those of the very reliable DIM. Close to cutoff the results of the DSM suggest, contrary to the results of some other methods, that, for this structure, there is no cutoff frequency. This is the correct result as the absence of any cutoff frequency, for the fundamental mode, is typical for perturbations in homogeneous media, for both one-dimensional and two-dimensional systems [14].

\section{Conclusions}

We have presented a simple scalar method to solve the wave equations for two-dimensional waveguides. The method is relatively fast with respect to the computation time and takes only very little computer storage if one is interested in the propagation constant only. The method gives results which are comparable with those of similar methods.

\section{ACKNOWLEDGMENT}

The author thanks his colleagues A. Driessen, P. V. Lambeck, and T. J. A. Popma from the optical group of the S\&A Institute of the University of Twente for helpful discussions.

\section{REFERENCES}

[1] K. Bierwirth, N. Schultz, and F. Arndt, "Finite-difference analysis of rectangular dielectric waveguide structures," IEEE Trans. Microwave Theory Tech., vol. MTT-34, pp. 1104-1113, 1986.

[2] D. De Zutter et al., "COST-216 comparison of different modeling techniques for longitudinally invariant integrated optical waveguides," Proc. IEE J., vol. 136, pp. 273-280, 1989.

[3] H. Diestel, "A method for calculating the guided modes of striploaded optical waveguides with arbitrary index profile," IEEE $J$. Quantum Electron., vol. QE-20, pp. 1288-1293, 1984.
[4] C. H. Henry and B. H. Verbeek, "Solution of the scalar wave equation for arbitrarily shaped dielectric waveguides by two-dimensional Fourier analysis,', J. Lightwave Technol., vol. 7, pp. 308-313, 1989.

[5] B. M. A. Rahman and J. B. Davies, "Finite-element analysis of optical and microwave waveguide problems," IEEE Trans. Microwave Theory Tech., vol. MTT-32, pp. 20-28, 1984.

[6] A. W. Snyder and J. D. Love, Optical Waveguide Theory. London: Chapman and Hall, 1983.

[7] G. Dahlquist and $\AA$. Björk, Numerical Methods. Englewood Cliffs, NJ: Prentice-Hall, 1974

[8] H. Kogelnik, in T. Tamir, Ed., Integrated Optics. New York: Springer, 1979.

[9] J. J. G. M. van der Tol and N. H. G. Baken, "Correction to effective index method for rectangular dielectric waveguides," Electron. Lett. vol. 24, pp. 207-208, 1988.

[10] D. A. Roberts and M. S. Stern, "Accuracy of method of moments and weighted index method,"' Electron. Lett., vol. 23, pp. 784-785, 1987.

[11] E. W. Kolk, N. H. G. Baken, and H. Blok, "Domain-integral equation of integrated-optical channel and ridge waveguides in stratified media," J. Lightwave Technol., to be published.

[12] J. E. Goell, "A circular-harmonic computer analysis of rectangular dielectric waveguides," Bell Syst. Tech. J., vol. 48, pp. 2133-2160, 1969

[13] A. Sharma, P. K. Mishra, and A. K. Ghatak, "Single-mode optical waveguides and directional couplers with rectangular cross-section: A simple and accurate method of analysis," J. Lightwave Technol. vol. 6, pp. 1119-1125, 1988.

[14] E. N. Economou, Green's Functions in Quantum Physics. (Springer Series in Solid-State Sciences, vol. 7). New York: Springer, 1983.

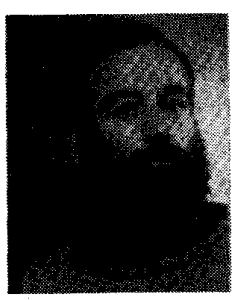

Hugo J. W. M. Hoekstra received the M.Sc. degree in experimental physics from the University of Amsterdam. Amsterdam. The Netherlands, in 1977. After being a teacher in high school, he began $\mathrm{Ph} . \mathrm{D}$. study on magnetooptical properties of solid transition-metal halides at the University of Groningen, where he received the Ph.D. degree in 1984 .

Between 1984 and 1988 he was a Postdoctoral Fellow in the field of surface science at the Univesities of Nijmegen and Groningen. In 1988 he joined the Optical Group of the Sensors and Actuators Institute of the University of Twente, Enschede, The Netherlands, where he is engaged in work on linear and nonlinear integrated optics. 